\title{
WiZard: a Program to Measure Cosmic-Ray Antiprotons and Positrons, and Search for Primordial Antimatter.
}

\section{R. L. GoLDEN}

Particle Astrophysics Laboratory, New Mexico State University - Las Cruces, NM

S. P. Ahlen and J. J. Beatty

Department of Physics, Boston University - Boston, MA

H. J. CRAWFord and P. J. LindSTRom

Space Sciences Laboratory, University of California - Berkeley, CA

J. F. ORMES and R. E. STReitmatTer

NASA Goddard Space Flight Center - Greenbelt, MD

C. R. Bower, R. M. HeInz and S. Mufson

Department of Physics, Indiana University - Bloomington, IN

T. G. GuZIK and J. P. WEFEL

Department of Physics and Astronomy, Louisiana State University - Baton Rouge, LA

S. A. STHEPHENS

Tata Institute for Fundamental Research - Bombay, India

J. H. Adams, K. E. Krombel and A. J. Tylka

Naval Research Laboratory - Washington, DC

M. Simon and K. D. Mathis

University of Siegen - Siegen, B.R.D.

P. PicozzA

Dipartimento di Fisica, Università di Roma *Tor Vergata» - Roma, Italia

G. BARBIELLINI

CERN - Geneva, Switzerland

Dipartimento di Fisica dell'Università - Trieste, Italia

G. BASini, F. Bongionno and M. RicCi

Laboratori Nazionali INFN - Frascati (Roma), Italia

\section{A. Codino}

Dipartimento di Fisica dell'Università - Perugia, Italia

INFN - Sezione di Perugia 
C. De Marzo and B. Marangelli

Dipartimento di Fisica dell'Università - Bari, Italia

INFN - Sezione di Bari

P. Galeotti

Laboratori di Cosmogeofisica del CNR - Torino, Italia

P. Spillantini and M. Bocciolini

Dipartimento di Fisica dell'Università - Firenze, Italia

INFN - Sezione di Firenze.

(Nuovo Cimento B, 105, 191 (1990))

PACS 98.80 - Cosmology.

PACS 95.55 - Atronomical instruments.

PACS 96.40 - Cosmic rays.

PACS 99.10 - Errata.

The name of one of the authors has been unluckily misprint. We publish here above the correct version, B. MARANGELLI instead of B. MANAGELLI, sincerely apologizing to the author. 